\title{
Tuning the properties of colloidal magneto-photonic crystals by controlled infiltration with superparamagnetic magnetite nanoparticles
}

\author{
PJ. Demeyer, M. Bloemen, T. Verbiest, and K. Clays \\ K.U.Leuven, Dept. of Chemistry, Celestijnenlaan 200D bus 2425, 3001 Heverlee, Belgium
}

\begin{abstract}
The performance of magnetic-field sensors and optical isolators is largely determined by the efficiency of the active materials. This efficiency could be dramatically increased by integrating Faraday materials in photonic crystals. For this purpose, monodisperse nanospheres were self-assembled into a colloidal photonic crystal and magnetic functionality was introduced by dipping the photonic crystal in a suspension containing superparamagnetic nanoparticles. Reflection and absorbance measurements of these magneto-photonic crystals revealed clear relationships between the time spent in suspension and the position and strength of the photonic band gap. When additional magnetic material was introduced, the band gap was red shifted and the strength of the band gap was decreased. Using Bragg's law and the Maxwell-Garnet approximation for effective media, the filling fraction of the magneto-photonic crystals was calculated from the observed red shift.
\end{abstract}

While superparamagnetic nanoparticles did confer magneto-optical properties to the photonic crystal, they also increased the absorption, which can be detrimental as the Faraday effect is measured in transmission. Therefore a trade-off exists in the optical regime between the amount of Faraday rotation and the absorption. By carefully controlling the filling fraction, this trade-off was investigated and optimized for photonic crystals with different band gaps. Both polystyrene and silica photonic crystals were filled with superparamagnetic nanoparticles. In case of the polystyrene photonic crystals, it was found that the maximum achievable filling fraction was influenced by the size of the polystyrene nanospheres. Smaller polystyrene nanospheres gave rise to smaller pore diameters and a faster onset of pore blocking when filled with superparamagnetic nanoparticles. As a result, the maximum achievable filling fraction was also lower. Pore blocking was found to be negligible in silica photonic crystals. Together with a higher mechanical strength, this makes silica photonic crystals more suited for the fabrication of colloidal magneto-photonic crystals.

In this paper, a nanoscale engineering approach is described to carefully control the filling fraction of magneto-photonic crystals. This allows fine-tuning the absorption and the position and strength of the photonic band gap. By tailoring the properties of magneto-photonic crystals, the means for application-specific designs and a better description of Faraday effects in 3D magneto-photonic crystals are provided.

Keywords: Photonic crystals, superparamagnetic nanoparticles, magneto-photonic crystals

\section{INTRODUCTION}

Modern demands for high speed technology for telecommunication and data processing have created the need for advanced materials and nanostructures allowing manipulation of electromagnetic radiation in the optical spectral range. Subsequent research has shown that photonic crystals provide excellent candidates to accommodate these needs. Photonic crystals are a relatively new class of materials in which the refractive index is periodic in 1, 2 or 3 dimensions, with the scale of periodicity being on the same length scale as the wavelength of light one wishes to influence. In analogy with semiconductors, this periodicity gives rise to a photonic band gap, i.e. a range of wavelengths for which there are no modes available in the direction of the periodicity. As a result, light with a wavelength coinciding with the photonic band gap cannot propagate in the direction of the periodicity and is selectively reflected. This principle has been successfully implemented in e.g. photonic crystal fibers. Another interesting property of photonic crystals is the anomalous dispersion that occurs close to the photonic band gap (i.e. at the band edge). This causes the group velocity of light to decrease considerably, allowing stronger interactions between the travelling light and the material of which the photonic crystal consists. ${ }^{[1]}$

When nonlinear optical materials are integrated into the photonic crystal structure, the slowing of light can give rise to a large enhancement of both reciprocal and non-reciprocal optical phenomena. In the case of magneto-photonic crystals, 
magnetic material is introduced into the photonic crystal, enhancing their intrinsic magneto-optical properties (e.g. Faraday rotation). As such, they have strong potential for applications in highly efficient and integrated optical isolators and very sensitive magnetic field sensors.

Preceding research has shown that the Faraday response is indeed strongly increased near the band edge in 1D and 2D photonic crystals. ${ }^{[2,3]}$ In colloidal 3D photonic crystal a smaller effect on the Faraday spectrum has been described but there is still significant room for improvement. ${ }^{[4,5]}$ Currently, the main issue is to introduce sufficient magnetic material in the 3D photonic crystal in a uniform and reproducible way, without absorbing too much light (the Faraday effect is measured in transmission). An additional trade-off exists between the amount of magnetic material in the 3D photonic crystal and the strength of the photonic band gap. As more magnetic material is introduced in the voids of the colloidal photonic crystal, the refractive index contrast is lowered, giving rise to a weaker and red shifted band gap. ${ }^{[1,6]}$ In this paper, a method is described to carefully control and fine-tune the amount of introduced magnetic material and hence the absorbance of the material and the position and strength of the photonic band gap.

\section{EXPERIMENTAL}

\subsection{Superparamagnetic magnetite nanoparticles}

Superparamagnetic magnetite nanoparticles were synthesized by thermal decomposition and subsequently silanized with methoxy(polyethyleneoxy)-propyltrimethoxysilane. ${ }^{[1,12]}$

\subsection{Magneto-photonic crystals from polystyrene nanospheres}

Polystyrene nanospheres (Interfacial Dynamics Corporation, Portland) with diameters of $170 \mathrm{~nm}$ (Coefficient of Variation $(\mathrm{CV})=2,3 \%)$ and of $220 \mathrm{~nm}(\mathrm{CV}=5,3 \%)$ were dissolved separately in MilliQ water (7 ml) at a concentration of $0,3 \mathrm{wt} \%$. The polystyrene nanoparticle suspensions were poured in a glass vial and put in an oven at $55^{\circ} \mathrm{C}$ together with glass slides $(18 \times 18 \mathrm{~mm}, 170 \pm 5 \mu \mathrm{m}$ thick) for convective self-assembly. After self-assembly, a short sintering step (5 min at $103^{\circ} \mathrm{C}$ ) was performed to improve the mechanical properties of the photonic crystals. Infiltration of the photonic crystals was achieved by repeated dipping of the photonic crystals in a suspension of superparamagnetic nanoparticles in ethanol $(5 \mathrm{mg} / \mathrm{ml})$ using a home-made mechanical dipping system at a dipping speed of $10 \mathrm{~cm}$ per minute. Each dipping step caused the sample to be in the suspension for 12 seconds. Between dippings, the ethanol was evaporated and the reflection spectrum of the sample was recorded.

\subsection{Magneto-photonic crystals from silica nanospheres}

Silica nanospheres of various sizes were synthesized following the Stöber-Bohn method ${ }^{[9]} .4 \mathrm{ml}$ of MilliQ water, $3 \mathrm{ml}$ of ammonia (25\%, Merck Chemicals, Germany) and $60 \mathrm{ml}$ of ethanol (99,9\%, VWR, United States) were mixed in a clean plastic vial with a magnetic stirrer. The plastic vial was placed inside a double-walled beaker, connected to a temperature controlled water tiller. After 30 minutes, $4 \mathrm{ml}$ of tetraorthosilicate (98\%, Acros organics, Belgium) was added under continuous stirring to initiate the reaction. After more than 3 hours, the resulting nanospheres were washed by centrifuging and resuspending them 3 times in ethanol. Consecutively, the silica nanospheres were poured in a glass vial at a concentration of 5,5 g/l in $7 \mathrm{ml}$ of ethanol. Two glass slides were placed vertically in each glass vial and the vials were placed in an oven at $37^{\circ} \mathrm{C}$ for $2-3$ days to allow convective self-assembly. The small amounts of ethanol that remain after self-assembly were removed by placing the photonic crystals in a second oven at $150^{\circ} \mathrm{C}$. Infiltration of the photonic crystals was achieved by repeatedly dipping of the photonic crystals in a suspension of superparamagnetic nanoparticles in ethanol $(5 \mathrm{mg} / \mathrm{ml})$. The photonic crystal remained in the suspension for 1 minute per dip. After each dip, the ethanol was evaporated and the absorbance spectrum was measured.

\subsection{Characterization tools}

Scanning Electron Microscopy (SEM) images were made using a XL30 FEG ESEM (Philips), Transmission Electron Microscopy (TEM) images were recorded with an EM900 (Zeiss). The reflectance and absorbance of the photonic crystals were measured using a Lambda 900 UV/VIS/NIR spectrometer (PerkinElmer). For the reflectance measurements, an additional accessory, a forked optical fiber and a home-made sample holder were used. All reflectance and absorbance measurements were done at normal incidence (perpendicular to the (111) plane). 


\section{RESULTS AND DISCUSSION}

\subsection{Colloidal suspensions and colloidal photonic crystals}

In a first series of experiments, photonic crystals were prepared by convective self-assembly of $170 \mathrm{~nm}$ or $220 \mathrm{~nm}$ polystyrene nanoparticles. After self-assembly, random hexagonal close-packed (Rhcp, closely related to fcc) crystal structures, were obtained (see Figure 1a), with the (111) plane parallel to the glass surface. The monocrystalline domains (roughly $100 \mu \mathrm{m} \times 100 \mu \mathrm{m}$ ) were separated by large cracks in the crystal structure, as is typical in colloidal photonic crystals. $^{[10]}$
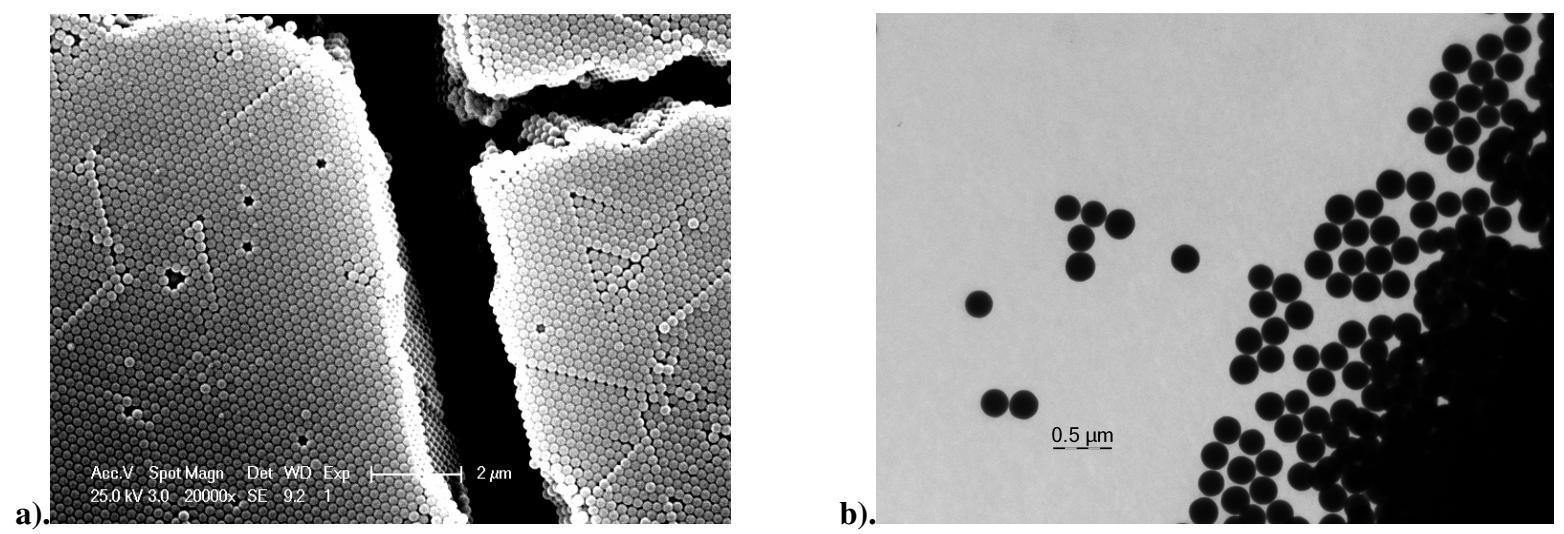

Figure 1: a) Typical SEM image of a colloidal photonic crystal with cracks and defects (220 nm polystyrene nanospheres). b) Typical TEM image of highly monodisperse silica nanospheres $(240 \mathrm{~nm})$.

In a second series of experiments, highly monodisperse silica nanospheres (see Figure 1b) with diameters of $180 \mathrm{~nm}, 220$ $\mathrm{nm}$ and $273 \mathrm{~nm}$ were synthesized and self-assembled into photonic crystals. The temperature is crucial during the synthesis of silica nanospheres as it determines the number of nucleation sites and hence the final size of the silica nanospheres. As the size of the silica nanospheres is directly proportional to the spectral position of the photonic band gap (see Section 3.2), the position of the photonic band gap could be freely chosen by adjusting the reaction temperature (see Figure 2a). The resulting silica photonic crystals have the same structural properties as the polystyrene photonic crystals described above.

Superparamagnetic magnetite nanoparticles were infiltrated into colloidal photonic crystals to enhance the magnetooptical properties. The synthesized superparamagnetic magnetite nanoparticles are very monodisperse and have a diameter of $9.3 \pm 1.6 \mathrm{~nm}$ (see Figure $2 \mathrm{~b}$ ). After synthesis, they were silanized to prevent nanoparticle aggregation. In this way, the uniformity of infiltration is improved and loss of superparamagnetism is avoided.
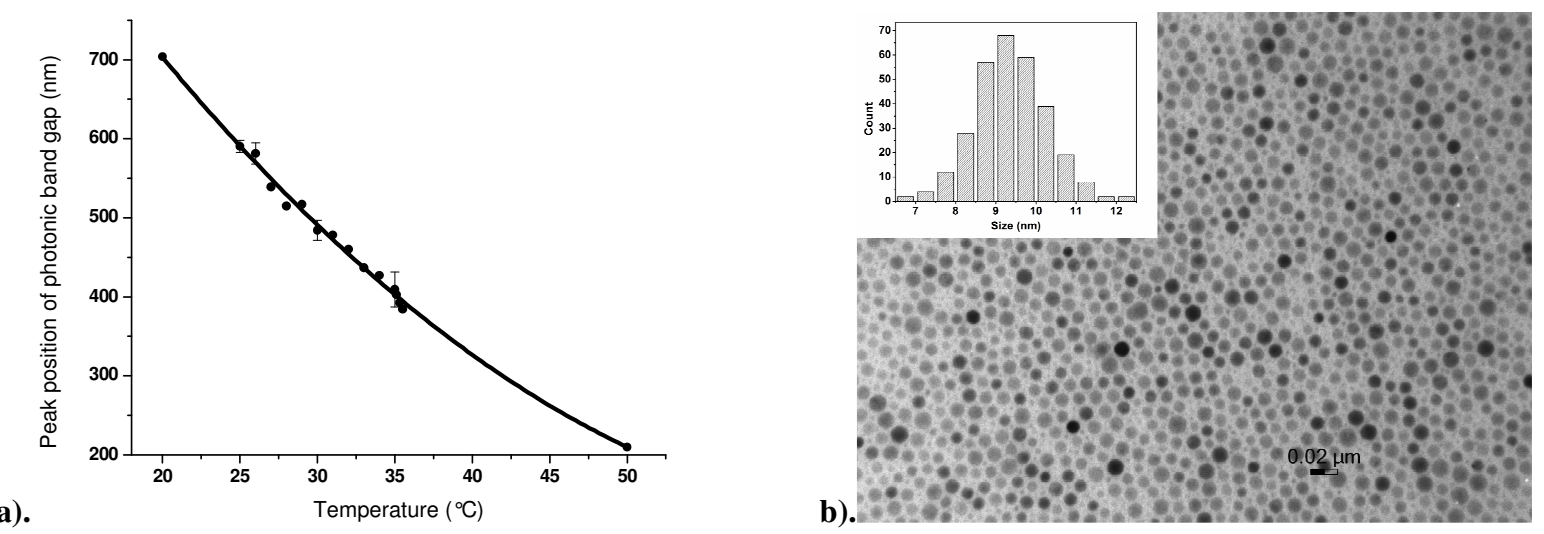

Figure 2: a) The spectral position of the photonic band gap strongly depends on the synthesis temperature for the silica nanospheres. b) TEM image of superparamagnetic magnetite nanoparticles and their size distribution (inset). 


\subsection{Optical characterization and determination of the filling fraction}

The spectral position of the photonic band gap in colloidal photonic crystals is given by the Bragg law for fcc closepacked structures at normal incidence ${ }^{[7]}$ :

$$
\lambda_{\max }=2 \sqrt{\frac{2}{3}} D \cdot n_{e f f}
$$

where $\lambda_{\max }$ is the spectral position of the band gap peak and D is the diameter of the nanospheres used for convective self-assembly. $n_{\text {eff }}$ is the effective refractive index and can be described by various effective media expressions. In this work, the Maxwell-Garnet equation was used for this purpose ${ }^{[8]}$ :

$$
\frac{n_{e f f}^{2}-1}{n_{e f f}^{2}+2}=\sum_{i} f_{i} \frac{n_{i}^{2}-1}{n_{i}^{2}+2}
$$

where $f_{i}$ are the volume fractions of the materials present in the effective medium and $n_{i}$ are their refractive indices. Before infiltration, the only materials present are silica or polystyrene, and the air in the void of the photonic crystals. As the refractive indices of these materials and their dispersion are well known, the combination of equation (1) and (2) allows calculating the average diameter of the nanospheres. The calculated diameters were in good accordance with those measured with SEM and TEM (data not shown).

Before infiltration, the refractive index contrast between the polystyrene or silica nanospheres and the air in the voids is responsible for the creation of the photonic band gap. When magnetic nanoparticles (high refractive index) are introduced, the average refractive index of the voids of the photonic crystal is gradually increased. As such, the refractive index contrast between the spheres and the voids is lowered and the photonic band gap is weakened and red shifted (see Figures 3 and 5). This behavior is in accordance with previously conducted modeling work described in ${ }^{[6]}$. The weakening and red shifting of the photonic band gap continues until the refractive index of the voids (air and magnetic nanoparticles) is equal to that of the nanospheres. When the filling factor is increased even further, the refractive index of the voids becomes higher than that of the nanospheres, leading to an increased refractive index contrast and an increasing peak intensity. ${ }^{[6]}$ Note that this inversion point was not reached during the experiments described in Sections 3.4 and 3.5.

The red shifting of the photonic band gap can be used to determine the filling fraction of magnetic nanoparticles, i.e. the volume percentage of the voids that is filled with magnetic material. Using the Bragg law, the peak maximum after (partial) infiltration can be related to an effective refractive index. The volume percentage of magnetic material in the total structure $\left(f_{M N P}\right)$ can subsequently be solved from the Maxwell-Garnet equations, which in this case takes the following form:

$$
\frac{n_{e f f}^{2}-1}{n_{e f f}^{2}+2}=f_{P S / S i O_{2}} \frac{n_{P S / S i O}^{2}-1}{n_{P S / S i O_{2}}^{2}+2}+f_{\text {air }} \frac{n_{\text {air }}^{2}-1}{n_{\text {air }}^{2}+2}+f_{M N P} \frac{n_{M N P}^{2}-1}{n_{M N P}^{2}+2}
$$

As the volume fractions of voids (26\%) and spheres $(74 \%)$ in a fcc crystal are known, the filling fraction (FF) could be obtained by:

$$
F F=\frac{f_{M N P}}{0.26}
$$




\subsection{Controlled filling of polystyrene photonic crystals with superparamagnetic nanoparticles}
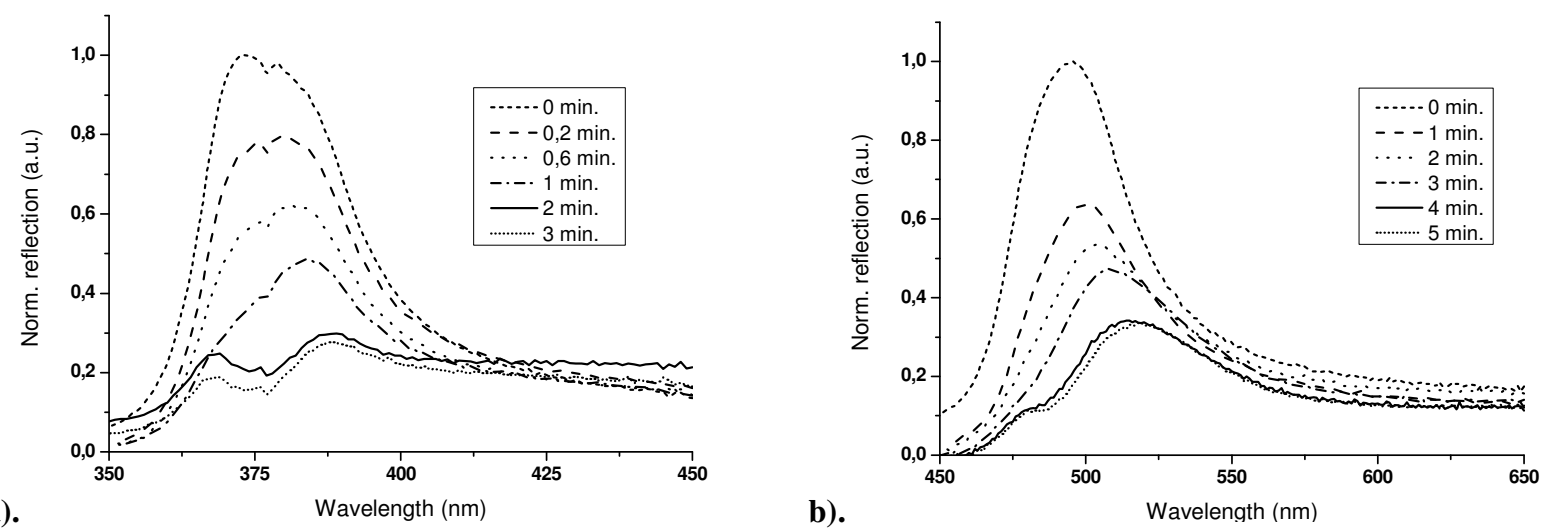

Figure 3: Normalized reflection spectra of the photonic crystals before and after infiltration. a) $220 \mathrm{~nm}$ polystyrene nanospheres, b) $170 \mathrm{~nm}$ polystyrene nanospheres.

When dipped in the suspension of superparamagnetic nanoparticles for longer times, both samples displayed a clear decrease in the peak intensity (fitted as a $1^{\text {st }}$ order exponential decay) and a gradual increase in the filling fraction (fitted as a Boltzmann curve). A stronger decrease in the peak intensity and a faster increase in the filling fraction were observed for the photonic crystal consisting of $170 \mathrm{~nm}$ polystyrene nanoparticles than for the one consisting of $220 \mathrm{~nm}$ polystyrene nanoparticles (see Figures $4 \mathrm{a}$ and $4 \mathrm{~b}$ ). As the voids are smaller in the photonic crystal with smaller spheres, the same amount of material will initially fill a higher volume percentage of the voids and therefore will also decrease the refractive index contrast faster. However, the pores are also smaller, leading to faster pore blocking when using the same size of magnetic nanoparticles. This is clearly visible in Figure $4 \mathrm{~b}$ as the $170 \mathrm{~nm}$ polystyrene nanoparticles already give rise to saturation while the curve of the $220 \mathrm{~nm}$ polystyrene nanoparticles is still in the linear regime. From these data it can be concluded that the use of smaller magnetic nanoparticles and/or larger polystyrene nanoparticles should lead to higher filling fractions. Unfortunately, the mechanical strength of the polystyrene photonic crystals was insufficient to continue the filling process for longer periods of time. Therefore, the more robust silica photonic crystals seemed more suitable candidates to fabricate colloidal magneto-photonic crystals.
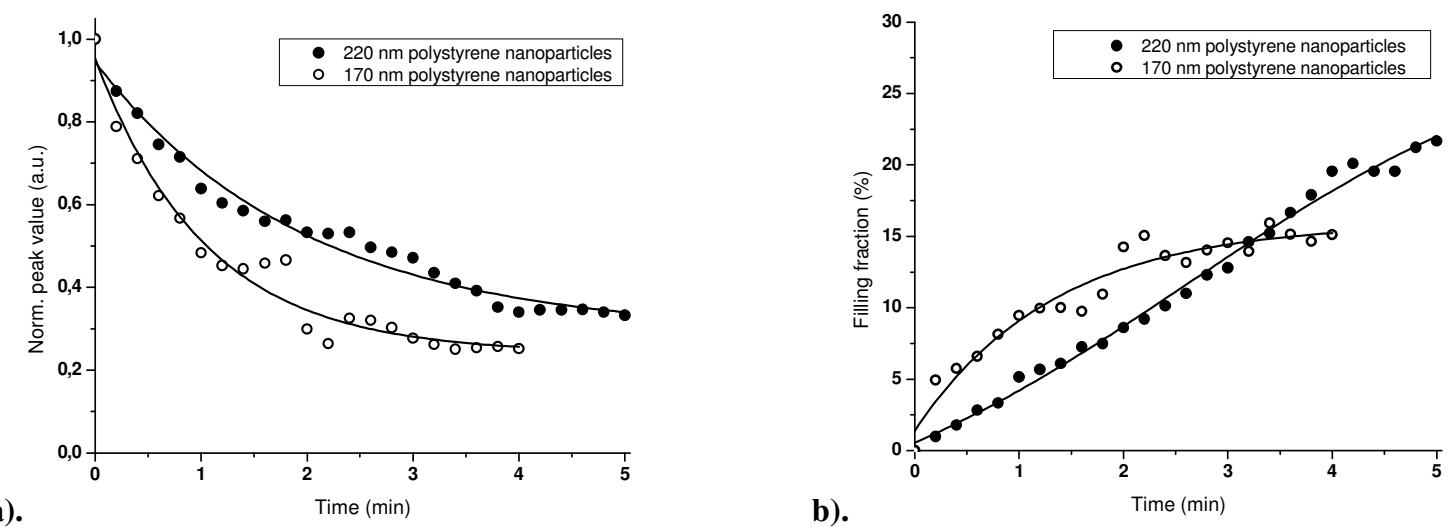

Figure 4: a) Normalized reflection peak values. b) Filling fractions calculated from the red shift of the reflection peak maxima.

\subsection{Controlled filling of silica photonic crystals with superparamagnetic nanoparticles}

In a second series of experiments, silica nanospheres of similar sizes as before were synthesized (180 nm and $220 \mathrm{~nm})$. Additionally, larger silica nanospheres $(273 \mathrm{~nm})$ were made to postpone the onset of pore blocking. Five photonic crystals were self-assembled from silica nanoparticles of each size and the filling process was followed optically. This time, the absorbance spectrum was measured instead of the reflection spectrum as the used equipment has a much higher signal-to-noise ratio in absorbance mode. The absorbance spectra were a superposition of the photonic band gap with the 
dispersive background caused by the absorption of the superparamagnetic nanoparticles and the silica nanospheres on one hand and by incoherent elastic scattering of the nanospheres on the other. The dispersive background was corrected for before further data analysis as it leads to an artificial blue-shifting of the photonic band gap. The rest of the data processing was done as described in 3.2 .
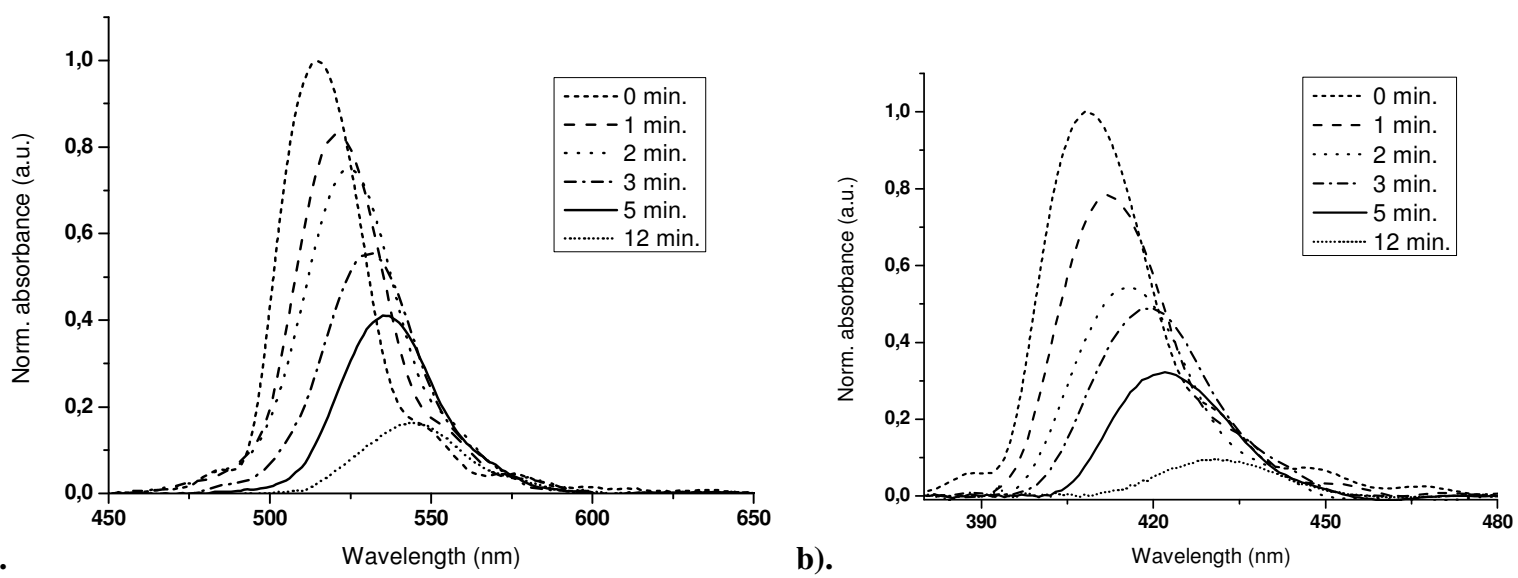

Figure 5: Normalized and baseline corrected absorbance spectra of the photonic crystals before and after infiltration. a) $220 \mathrm{~nm}$ silica nanospheres, b) $273 \mathrm{~nm}$ silica nanospheres.

As can be seen in Figure 5, all samples again displayed a strong decrease in the peak intensity and a red shifting of the photonic band gap. The $180 \mathrm{~nm}$ nanospheres gave rise to a faster decrease in the peak intensity than the $273 \mathrm{~nm}$ nanospheres (see Figure 6a), which is consistent with the results obtained in 3.3. No conclusions can be drawn concerning the $220 \mathrm{~nm}$ silica nanospheres as standard deviations overlap. Following the filling fraction as function of the time spent in suspension gave rise to saturation curves for all samples, with a maximum achievable filling fraction around $25 \%$. On average, the $220 \mathrm{~nm}$ silica nanospheres had a faster increase in the filling fraction than the $273 \mathrm{~nm}$ silica nanoparticles (see Figure 6b). Surprisingly, we did not see a dependence of the maximum achievable filling fraction on the size of the used silica nanospheres. The saturation behavior of the $180 \mathrm{~nm}$ nanosphere had a strong sample-to-sample variation (data not shown). Both the faster decrease in the peak intensity and the faster increase in filling fraction for smaller particles are consistent with the results from polystyrene photonic crystals. This was explained by the fact that the same amount of material will fill a higher volume percentage of smaller voids.
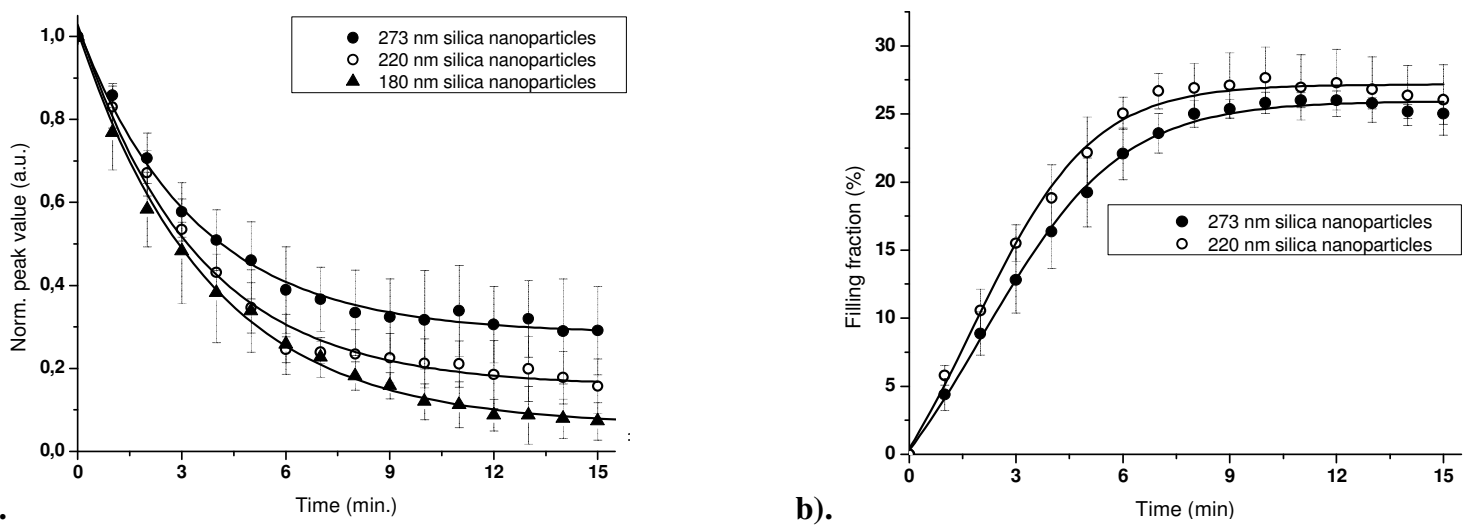

a).

Figure 6: a) Normalized absorbance peak values. b) Filling fractions calculated from the red shift of the absorbance peak maxima.

The absence of a size-dependence of the maximum achievable filling fraction indicates that pore blocking seems to be negligible in silica photonic crystals. This striking difference between the polystyrene and silica photonic crystals can 
likely be attributed to the different natures of the surface interactions. The factor that is currently limiting the achievable filling fraction might be the concentration of superparamagnetic nanoparticles in suspension. Since higher concentrations of superparamagnetic nanoparticles are certainly achievable, we believe that there is still room for further improvements.

\section{CONCLUSION}

A method was described to carefully control and fine-tune the amount of magnetic material and hence the absorbance of magneto-photonic crystals and the position and strength of photonic band gaps. The filling procedure can easily be followed optically. In case of polystyrene photonic crystals, it was found that maximum achievable filling fraction was influenced by the size of the polystyrene nanospheres. This was attributed to an earlier onset of pore blocking when using smaller polystyrene nanospheres. Pore blocking was found to be negligible in silica photonic crystals. Together with a higher mechanical strength, this makes silica photonic crystals more suited for the fabrication of colloidal magneto-photonic crystals. The described tailoring of the properties of magneto-photonic crystals will allow applicationspecific designs and a better description of magneto-optical effects in 3D magneto-photonic crystals.

\section{REFERENCES}

[1] Joannopoulos J.D., Johnson S.G., Winn J.N., Meade R.D., [Photonic Crystals: Molding the flow of light], Princeton University Press, Princeton, 94-154 (2008)

[2] Inoue M.. Fujikawa R., Baryshev A., Khanikaev A., Lim P.B., Uchida H., Aktsipetrov O., Fedyanin A., Murzina T, Granovsky A., "Magnetophotonic crystals”, J. Phys. D: Appl. Phys., 39(8), 151-161 (2006)

[3] Zvezdin A.K., Belotov V.I., "Magnetooptical Properties of Two Dimensional Photonic Crystals”, Eur. Phys. J. B, 37(4), 479-487 (2004)

[4] Koerdt C., Rikken G.L.J.A., Petrov E.P., "Faraday effect of photonic crystals”, Appl.Phys.Lett., 82(10), 1538-1540 (2003)

[5] Pavlov V.V., Usachev P.A., Pisarev R.V., Kurdyukov D.A., Kaplan S.F., Kimel A.V., Kirilyuk A., Rasing Th., "Enhancement of optical and magneto-optical effects in three-dimensional opal $/ \mathrm{Fe}_{3} \mathrm{O}_{4}$ magnetic photonic crystals", Appl. Phys. Lett., 93(7), 072502 (2008)

[6] Libaers W., Kolaric B., Vallée R.A.L., Wong J.E., Wouters J., Valev V.K., Verbiest T., Clays K., "Engineering colloidal photonic crystals with magnetic functionalities", Colloids and Surfaces A:Physicochem. Eng. Apects, 339, 13-19 (2009)

[7] Míguez H., López C., Meseguer F., Blanco A., Vázquez L., Mayoral R., Ocaña M., Fornés V., Mifsud A., "Photonic crystal properties of packed submicrometric $\mathrm{SiO}_{2}$ spheres", Appl. Phys. Lett., 71(9), 1148-1150 (1997)

[8] Garnett J.C.M., "Colours in Metal Glasses and in Metallic Films”, Philos. Trans. R. Soc. London Ser. A, 203, 385$420(1904)$

[9] Stöber W., Fink A., Bohn E., "Controlled growth of monodisperse silica spheres in the micron size range", J. Colloid and interface science, 26(1), 62-69 (1968)

[10] González-Urbina L., Baerts K., Kolaric B., Pérez-Moreno J., Clays K., "Linear and nonlinear optical properties of colloidal photonic crystals", Chem. Rev. , upcoming issue (2012)

[11] Park J., An K., Hwang Y., Park J., Noh H., Kim J, Park J., Hwang N., Hyeon T.,’Ultra-large-scale syntheses of monodisperse nanocrystals", Nature mat., 3, 891-895 (2004)

[12] Larsen E., Nielsen T., Wittenborn T., Birkedal H., Vorup-Jensen T., Jakobsen M., Østergaard L., Horsman M.R., Besenbacher F., Howard K.A., Kjems J., "Size-dependent accumulation of PEGylated silane-coated magnetic iron oxide nanoparticles in Murine tumors", ACS Nano, 3(7), 1947-1951 (2009) 